\title{
Interactive comment on "Contrasting seasonal changes in total and intense precipitation in the European Alps from 1903 to 2010" by Martin Ménégoz et al.
}

\section{Anonymous Referee \#3}

Received and published: 6 April 2020

\section{Review of Menegoz et al.}

In this manuscript the authors present a set of simulations to study if their model can reproduce the observed trends of precipitations over the alpine region during the last century. For that purpose, they set their regional model domain to run at a high resolution so that the orography and subsequent feedbacks can be more accurately represented. They then compare their trends with observations from a set of rain gauges over Switzerland and argue about which of these trends can be represented. I think the paper is of interest to the community and I recommend its publication after some revisions have been performed. 
-Sections 4.1 and 4.2: The most characteristic pattern of your model is the drying on the Po valley over summer. This is most likely related to the evolution of convective processes in your model as the climate warms. The observations in the southern part of Switzerland do not capture this trend at all, therefore bringing doubts about the physical reliability of the simulated signal. I understand that it might be complicated to get station data over the Po valley, however some datasets like EOBS are open and provide data since 1950. You should compare at least if in these datasets there is also a similar signal to what the model predicts. In case that is not observed one should consider whether if the observed trends are physical or a simple artifact coming from the parameterization of convective processes in your model.

-Section 4.3: It would be a great addition to the paper to compare the trends observed in extreme precipitation with the Clausius-Clapeyron relation, or at least give an estimate on how much they change per degree of warming.

Small comments:

-L56 Name the reasons: snow cover feedback etc... -L100 Mention the typical timescales of the NAO -L183: $7 \mathrm{~km}$ horizontal resolution on the gray zone of convection, is your parameterization prepared for running at those scales? If it is not scale dependent, did you test the behavior of the model when switching it off? At these scales, convection should appear already in a quite nice form, the use of a non-scale dependant parameterization might do more harm than good to the dynamics of the model. Perhaps in the future you should consider running a similar simulation turning off the parameterization of deep convection. I understand that this might be beyond the scope of this study, but you should mention that the model will likely be subject to some of the recursive biases of parameterized convection such as too frequent and too light precipitation spells. -L206: show the domain in a plot with the orography plotted and showing the size of your relaxation zone and the different analysis areas (SA, NWA, NEA). -L210: what is the resolution of ERA-20C? I think you should specify somewhere that the use of a regional model at such resolution is necessary to capture

Printer-friendly version

Discussion paper 
the spatial heterogeneity of the orography. Otherwise people might wonder why did you not simply use the reanalysis for looking at the trends. -L337: It is interesting to note that the large amounts of precipitation measured during summer at mid altitudes ( $\sim 6-8 \mathrm{~mm} /$ day; $500-1500 \mathrm{~m}$ ) cannot be predicted by the model. I guess these are likely stations strongly affected by convective precipitation, as the bias does not appear in winter. I wonder if this might happen due to including too much convective mixing in the atmosphere by your convective parameterization, therefore making precipitation to be too light where it should be much more stronger and intense. -L431: I think you mean Figure 4a? -L428-434: There are too many indexes used here that have not being presented before, some of them do not have names that make them easy to identify what they mean (SDII, STP, MNWS. . ), you should rewrite this part presenting the indexes before you analyze their trends. You should consider also a better naming for the indexes, SDII could just be Idaily, STP just Pseason etc. . -L445: I do not agree that the model and the observations are consistent. The observations tend to show a very weak signal if any, while the model specially in the southern part shows a very negative signal that is not captured by the southernmost stations at all.

-L540: As I mention in the first comment one should check if the trend in the Po valley has been observed. This is a very important question. The climate projections from the EURO-CORDEX ensemble show a similar behavior for the future climate (decrease in mean precipitation explained by decrease in frequency). If this behavior has not being measured in the observations one would wonder about the reliability of these projections, which is indeed a very important result. This is critical as the use of a convective parameterization biases very strongly the precipitation frequency of the models, therefore it might be just the parameterization overreacting to a perturbation in temperature.

-Table 1: I like the idea of explaining all the indexes in a table, but I think the description of the indexes should also appear in the text at least the first time they are used.

Printer-friendly version

Discussion paper

Interactive comment on Hydrol. Earth Syst. Sci. Discuss., https://doi.org/10.5194/hess-2019- 
690, 2020.

HESSD

Interactive

comment

Printer-friendly version

Discussion paper

(c) 